\title{
Bilateral visual outcomes and service utilization of patients treated for 3 years with ranibizumab for neovascular age-related macular degeneration
}

This article was published in the following Dove Press journal:

Clinical Ophthalmology

08 April 2014

Number of times this article has been viewed

\section{Randhir Chavan ${ }^{1, *}$ \\ Swati Panneerselvam 1,* \\ Parul Adhana' \\ Nirodhini Narendran' \\ Yit Yang ${ }^{1,2}$}

'Eye Infirmary, New Cross Hospital, Royal Wolverhampton Hospitals NHS Trust Wolverhampton,

West Midlands, UK; ${ }^{2}$ Faculty of Life and Health Sciences, Aston University, Birmingham, UK

*These authors contributed equally to this paper
Correspondence: Randhir Chavan Eye Infirmary, New Cross Hospital, Royal Wolverhampton Hospitals NHS Trust, Wednesfield Road, Wolverhampton, WVI0 0QP, UK Tel +44 1902695824

Fax +44 I902695 84I

Email randhir.chavan@doctors.org.uk
Background: The aim of this study was to describe bilateral visual outcomes and the effect of incomplete follow-up after 3 years of ranibizumab therapy for neovascular age-related macular degeneration. Secondarily, the demands on service provision over a 3-year period were described.

Methods: Data on visual acuity, hospital visits, and injections were collected over 36 months on consecutive patients commencing treatment over a 9-month period. Visual outcome was determined for 1) all patients, using last observation carried forward for missed visits due to early discontinuation and 2) only those patients completing full 36-month follow-up.

Results: Over 3 years, 120 patients cumulatively attended hospital for 1,823 noninjection visits and 1,365 injection visits. A visual acuity loss of $<15$ letters (L) was experienced by $78.2 \%$ of patients. For all patients $(n=120)$, there was a mean loss of $1.68 \mathrm{~L}$ using last observation carried forward for missing values. Excluding five patients who died and 30 who discontinued follow-up, mean gain was $1.47 \mathrm{~L}$. In bilateral cases, final acuity was on average $9 \mathrm{~L}$ better in second eyes compared to first eyes. Also, $91 \%$ of better-seeing eyes continued to be the better-seeing eye. Conclusion: We have demonstrated our approach to describing the long-term service provision and visual outcomes of ranibizumab therapy for neovascular age-related macular degeneration in a consecutive cohort of patients. Although there was a heavy burden with very frequent injections and clinic visits, patients can expect a good level of visual stability and a very high chance of maintaining their better-seeing eye for up to 3 years.

Keywords: visual acuity, real-world data, long term follow up, lucentis

\section{Introduction}

In the National Health Service of the UK, ranibizumab (Lucentis ${ }^{\circledR}$; Genentech, Inc., South San Francisco, CA, USA) monotherapy was recommended for patients with neovascular age-related macular degeneration (NV-AMD) by the National Institute of Clinical Excellence (NICE) in August 2008. ${ }^{1}$ The efficacy and safety of ranibizumab has been established by well-designed studies with endpoint analyses at 12 or 24 months. $^{2-6}$ There is evidence to show that the initial treatment benefit can be maintained for longer periods of between 3 to 7 years from the first treatment, ${ }^{7-11}$ but due to the high dropout rate in almost all published long-term studies and the potential selection bias in prospective clinical trial extension studies, it can be difficult to demonstrate the true, long-term treatment benefits and the burden of repeated injections over periods of 3 years or longer. The National Health Service setting in the UK is ideal for evaluating such parameters in a population that is uniformly managed, as up until very recently, ranibizumab has been used almost exclusively for NV-AMD, and decisions on 
retreatment are not influenced by patients' ability to pay. Our primary objective was to provide an accurate description of visual outcomes in a consecutive and representative cohort of patients 3 years after commencing ranibizumab therapy, taking into account the impact of incomplete follow-up on estimates of visual acuity outcomes. Secondary objectives were to describe the involvement of fellow eyes, maintenance of vision in better-seeing eyes, and the utilization of resources in terms of the number of injection and noninjection visits. It is envisaged that this unique report can be of value to clinicians, commissioners, and managers in highlighting the importance of accurate accountability of patient follow-up for the purpose of future audit comparisons and benchmarking.

\section{Materials and methods Setting}

Patients from a catchment population of about 850,000 suspected of having NV-AMD were referred to a single-site, fast track, treatment service for logMAR visual acuity test, biomicroscopy, optical coherence tomography (OCT), and fluorescein angiography (FA) +/- indocyanine green angiography if there were clinical features of polypoidal vasculopathy. Confirmation of NV-AMD was based on presence of drusen or age-related changes in the retinal epithelium on OCT and late-phase leakage on FA of subfoveal choroidal neovascularization with either classic, occult, or retinal angiomatous proliferative components. Eyes with NV-AMD and visual acuity of 6/12 to 6/96 were considered to satisfy the NICE guidance criteria ${ }^{1}$ and would receive a loading dose of three injections followed by retreatment on a pro re nata basis. Using a two-stop model, repeat injections were scheduled shortly after monitoring visits if lesions were found to be active. As much as possible, monitoring visits were scheduled every month. A treat and extend regimen was not practiced in our department.

Retreatment decision was OCT-guided and was performed until no further reduction in intra or subretinal fluid could be achieved. In accordance with retreatment protocols used in earlier ranibizumab pro re nata studies such as the PRONTO (Prospective optical coherence tomography imaging of patients with neovascular AMD treated with intraocular ranibizumab) study, ${ }^{12}$ we did not specifically continue repeat injections to attempt to resolve pigment epithelial detachments which were not associated with presence of subretinal or intraretinal fluid on OCT. Patients could be discharged from follow-up in the treatment program if they had not required retreatment in over 12 months or if it was felt that benefit could not be gained due to extensive macular damage.

\section{Patient selection and data analysis}

In line with our hospital policy, ethics committee approval for retrospective data collection studies was not required. Consecutive patients entering the treatment program between April 1, 2009 and December 31, 2009 were identified from an accurate injection database.

Patients were excluded from this survey if they had no follow-up after baseline injection or were recruited into a clinical trial. Eyes were excluded if they had any photodynamic therapy (PDT), lesions secondary to non-AMD causes, serous detachments of pigment epithelium, peripapillary lesion, or choroidal polyps.

Data were collected on patients' age and sex, laterality of treatment, lesion type, visual acuities, central retinal thickness (CRT), and number of FAs, OCTs, and treatment and nontreatment visits up to or nearest to month 36 or the final visit if follow-up was discontinued early. The reason for discontinuation was noted for each case.

First-treated eyes were defined as any eye treated at baseline (both eyes were included if they were simultaneously treated at baseline). Treated second, fellow eyes were defined as any eye treated at subsequent visits to the first injection visit. Visual outcome data were determined at baseline and at yearly time points in terms of mean levels (number of Early Treatment Diabetic Retinopathy Study letters, [L]), mean change, proportions who gained and lost $15 \mathrm{~L}$, and proportions in strata of $<25 \mathrm{~L}, 25-50 \mathrm{~L}, 50-75 \mathrm{~L}$, and $>75 \mathrm{~L}$. Last observation carried forward (LOCF) was used for missing values for those patients who had not died but were discharged early (full-analysis set).

To provide an indicator of overall visual outcomes at each time point, visual acuities were described by firstly analyzing all treated first and second eyes together and secondly by describing the visual outcomes in second eyes only.

To demonstrate the effect of using LOCF on reporting visual outcomes in real-world studies, where single samples or cohorts are used, we analyzed visual outcomes three times using different analysis sets. The first set was the per-protocol set, which contained patients who had full 3 year follow-up. The second set was the LOCF set, which contained patients who had not died but did not complete 3 year follow-up, and a third, full-analysis set, containing all patients. Values of visual outcomes over 3 years in these three analysis sets were tabulated for cross comparison without statistical testing to demonstrate the effects of using and not using LOCF.

To evaluate the impact of treatment on overall visual function, we compared the visual acuities at month 36 of treated and untreated eyes, and also determined the proportion of 
patients who had maintenance of vision in their better-seeing treated eyes.

Finally, to evaluate the anatomical effect of treatment, CRT values were compared between baseline and yearly time points and final visits in first-treated eyes.

\section{Results}

\section{Patients, eyes, visits, injections, and follow-up}

Over 9 months, 150 patients were identified, of whom 22 patients entered a clinical trial (IVAN [Inhibition of VEGF in age-related choroidal neovascularization] study), ${ }^{5}$ and another eight patients were excluded from analysis due to the following reasons: 1) lack of any follow-up after first injection (three), 2) prior PDT (two), 3) pathological myopia (two), and 4) peripapillary choroidal neovascularization (one). Of the remaining 120 patients, mean age $\pm \mathrm{SD}$ (standard deviation) was $81.76 \pm 6.71$ (range: 65-95) years, and 75 (62.5\%) were female. At baseline, all 120 patients had an OCT scan, $112(93 \%)$ had FA, and four (3\%) had indocyanine green angiography. The lesion types were occult $67 / 120$ (56\%), classic containing 46/120 (38\%), and retinal angiomatous proliferative 7/120 (6\%). One hundred and seventeen had at least 1 year follow-up, 107 had at least 2 year follow-up, and 85 had at least 3 year follow-up. The data collection period covered a total of 1,823 noninjection visits and 1,365 injection visits in our two-stop system (mean of 15.33 injections per patient, six in the first year, four in the second year, and four in the third year. Bilateral involvement occurred in four patients from baseline and another 14 patients required injections to second fellow eyes during follow-up. Therefore, we collected data on 124 first eyes, 14 second fellow eyes, and 102 untreated eyes. Out of 120 patients with 124 first-treated eyes receiving injections at baseline (full-analysis set), five died and $30 \mathrm{did}$ not complete 3 year follow-up, but 89 first study treated eyes of 85 patients $(71 \%)$ did complete 3 year follow-up (per-protocol set). Table 1 shows the numbers of patients, eyes, treatment visits, nontreatment visits, and discontinuations and their reasons.

\section{Visual outcome}

Visual outcomes for all treated eyes at each yearly time point are shown in Table 2. Baseline mean visual acuity was $52.24 \mathrm{~L}$ ( $\mathrm{SD} \pm 14.69 \mathrm{~L})$. At 36 months mean visual acuity was $51.01 \mathrm{~L}$ (SD $\pm 19.46 \mathrm{~L})$; mean change was $-1.68 \mathrm{~L}$ (SD $\pm 17.76 \mathrm{~L})$. Proportion of eyes with $<15$ L loss was $78.2 \%$, proportion of eyes with $>15 \mathrm{~L}$ gain was $16.80 \%$, proportion of treated eyes with $>75 \mathrm{~L}$ was $10.08 \%$, and proportion with $<25 \mathrm{~L}$ was $13.44 \%$. The percentage of patients in the four visual acuity strata at various time points for all patients completing 3 year follow-up are shown in Figure 1.

During the study period, 14 patients had treatment to second fellow eyes. These 14 eyes had mean baseline visual acuity of $59.28 \mathrm{~L}(\mathrm{SD} \pm 15.07 \mathrm{~L})$, which was better than first-treated eyes, indicating earlier presentation or diagnosis. By the time the first eyes had reached month 36,

Table I Numbers of patients' eyes exposed to treatment and follow-up

\begin{tabular}{|c|c|c|c|}
\hline$n=120$ & $\begin{array}{l}\text { Year I } \\
0-12 \text { months }\end{array}$ & $\begin{array}{l}\text { Year } 2 \\
\text { 13-24 months }\end{array}$ & $\begin{array}{l}\text { Year } 3 \\
\text { 25-36 months }\end{array}$ \\
\hline Number died $=5$ & I & I & 3 \\
\hline Number of patients in full-analysis set & 119 & 118 & 115 \\
\hline Number lost to follow-up $=30$ & 2 & 9 & 19 \\
\hline \multirow[t]{4}{*}{ Reasons for drop outs } & $F(n=2)$ & $F(n=I)$ & $F(n=I)$ \\
\hline & & $D(n=I)$ & $D(n=7)$ \\
\hline & & I $(n=7)$ & $I(n=10)$ \\
\hline & & & $T(n=I)$ \\
\hline Number of patients remaining at end of year & 117 & 107 & 85 \\
\hline Number of second eyes commencing treatment & 13 & 18 & 18 \\
\hline Number of first and second eyes actively treated in program & 130 & 125 & 103 \\
\hline Mean number of total visits (range) & $12.30(7-18)$ & $10.61(3-18)$ & $11.47(1-17)$ \\
\hline Mean number of injection visits (range) & $5.87(I-I I)$ & $4.06(0-10)$ & $4.21(0-11)$ \\
\hline Mean number of review visits (range) & $6.42(3-I I)$ & $6.54(2-12)$ & $7.25(1-13)$ \\
\hline Per-protocol set & 85 & 85 & 85 \\
\hline Mean number of total visits (range) & $12.87(9-18)$ & II.47 (3- I8) & II.47 (I-I7) \\
\hline Mean number of injection visits (range) & $6.42(1-11)$ & $4.69(0-10)$ & $4.21(0-11)$ \\
\hline Mean number of review visits (range) & $6.44(3-11)$ & $6.77(2-10)$ & $7.25(1-13)$ \\
\hline
\end{tabular}

Abbreviations: D, discharged due to no recurrence > I year; F, failed to attend; I, irreversible macular damage due to fibrosis or retinal pigment epithelial rip; T, transferred to other unit. 
Table 2 Details of visual outcomes in all treated eyes

\begin{tabular}{|c|c|c|c|}
\hline & Year I & Year 2 & Year 3 \\
\hline Number of patients & 119 & 118 & 115 \\
\hline \multirow[t]{2}{*}{ Number of treated eyes } & I 23 (4 bilateral & 122 & 119 (LOCF in 34 eyes) \\
\hline & from baseline included) & & \\
\hline Baseline VA & $52.24 \pm 14.69$ & $52.44 \pm 14.60$ & $52.89 \pm 14.63$ \\
\hline Mean VA at end of year & $51.01 \pm 19.46$ & $49.74 \pm 21.18$ & $51.15 \pm 21.09$ \\
\hline Mean change in VA from baseline & $-1.34 \pm 15.34$ & $-2.35 \pm 17.35$ & $-1.68 \pm 17.76$ \\
\hline Percentage with $<15 \mathrm{~L}$ loss & 84.56 & 80.33 & 78.2 \\
\hline Percentage with $>15 \mathrm{~L}$ gain & 13 & 12.29 & 16.80 \\
\hline Percentage with $<25 \mathrm{~L}$ & 13.82 & 13.11 & 13.44 \\
\hline Percentage with $>75 \mathrm{~L}$ & 9.75 & 9.01 & 10.08 \\
\hline Subset of second eyes affected at end of year & 9 & 14 & 14 \\
\hline Baseline VA & $64 \pm 12.14$ & $61 \pm 13.16$ & $59.28 \pm 15.07$ \\
\hline Mean VA at end of year & $66.44 \pm 15.39$ & $62.85 \pm 11.71$ & $56.92 \pm 16.55$ \\
\hline Mean change in VA from baseline & $2.44 \pm 6.48$ & $2 \pm|1.0|$ & $-2.35 \pm 11.64$ \\
\hline
\end{tabular}

Abbreviations: LOCF, last observation carried forward; VA, visual acuity; L, Early Treatment Diabetic Retinopathy Study letters.

the final mean visual acuity of second eyes was $56.92 \mathrm{~L}$ ( $\mathrm{SD} \pm 16.55 \mathrm{~L}$ ) (mean change $-2.35 \pm 11.64 \mathrm{~L}$ ), which was approximately $9 \mathrm{~L}$ better than the mean 36 month acuity of first eyes. The outcomes at yearly time points of second eyes are shown in Table 2.

Table 3 illustrates the different visual outcomes at the end of year 3 obtained by using the per-protocol set or the full-analysis set. In the full-analysis set, LOCF was used for missing values from 30 eyes of 30 patients who discontinued follow-up. These eyes had poorer outcome, with a mean loss of visual acuity from baseline to end of year 3 of $11.03 \mathrm{~L}$ ( $\mathrm{SD} \pm 20.29 \mathrm{~L}$, range $-63 \mathrm{~L}$ to $21 \mathrm{~L}$ ). One patient had visual loss of $63 \mathrm{~L}$ after the third injection. Removing this outlier made minimal difference to the overall estimate of mean change in visual acuity at month $36(-1.16 \pm 15.54 \mathrm{~L})$. In the per-protocol set, when only the 89 eyes of 85 patients who had full 3 years of follow-up were included, the visual outcomes appeared better. The mean visual acuity improved by $1.47 \mathrm{~L}(\mathrm{SD} \pm 15.60 \mathrm{~L})$ from $53.75 \mathrm{~L}(\mathrm{SD} \pm 13.59 \mathrm{~L})$ at baseline to $55.14(\mathrm{SD} \pm 17.97 \mathrm{~L})$ at the end of year 3. Outcomes

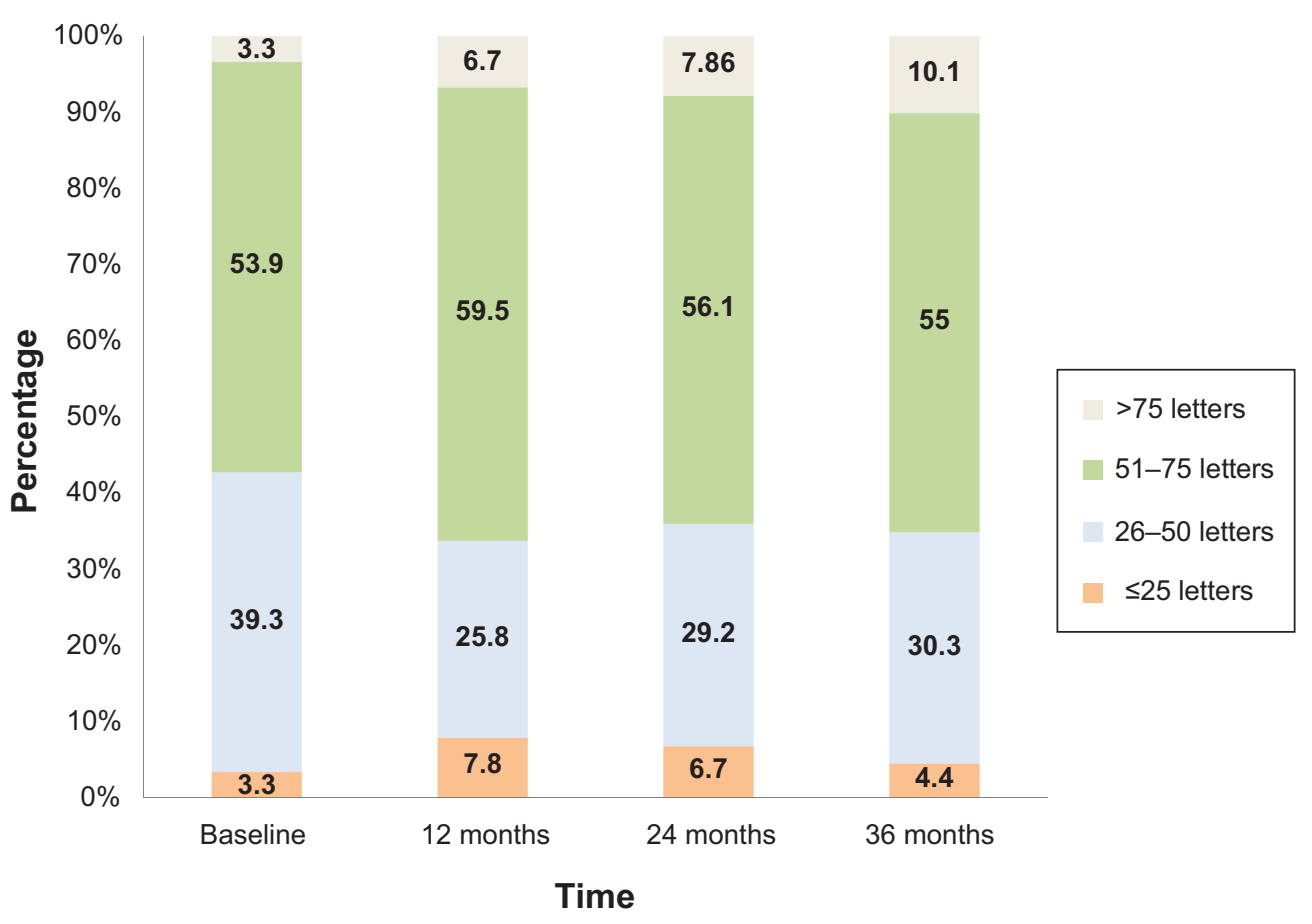

Figure I Distribution of patients who have completed three year follow-up across four strata of visual acuity at yearly time points. 
Table 3 Visual outcome parameters from baseline to end of year 3 in three different analysis sets

\begin{tabular}{llll}
\hline & $\begin{array}{l}\text { Per-protocol } \\
\text { set } \mathbf{n = 8 9}\end{array}$ & $\begin{array}{l}\text { LOCF } \\
\text { set } \mathbf{n = 3 0}\end{array}$ & $\begin{array}{l}\text { Full analysis set } \\
\text { (per protocol + LOCF) } \\
\mathbf{n}=\mathbf{I I 9}\end{array}$ \\
\hline VA at baseline mean \pm SD letters & $53.75 \pm 13.59$ & $50.33 \pm 17.10$ & $52.89 \pm 14.63$ \\
VA at end of year 3 mean \pm SD letters & $55.14 \pm 17.97$ & $39.3 \pm 24.86$ & $51.15 \pm 21.09$ \\
Mean VA change \pm SD letters & $+1.47 \pm 15.60$ & $-11.03 \pm 20.29$ & $-1.68 \pm 17.76$ \\
$<15$ letter loss (\%) & 84.26 & 60 & 78.2 \\
$\geq 15$ letter loss (\%) & 15.74 & 40 & 21.8 \\
$\geq I 5$ letter gain (\%) & 19.10 & 10 & 16.80 \\
\hline
\end{tabular}

Abbreviations: LOCF, last observation carried forward; SD, standard deviation; VA, visual acuity

in terms of proportions were also better: the proportion with $<15 \mathrm{~L}$ loss was $84.26 \%$, the proportion with $>15 \mathrm{~L}$ gain was $19.10 \%$, the proportion with $>50 \mathrm{~L}$ was $65 \%$, the proportion with $>75 \mathrm{~L}$ was $10 \%$, and the proportion with $<25 \mathrm{~L}$ was only $4.49 \%$.

\section{Prognosis of better-seeing eyes and never-treated fellow eyes}

In the per-protocol set with complete 3 year follow-up, 89 eyes of 85 patients were treated at baseline, and 81 fellow eyes never received treatment. The treated eyes were the better-seeing eyes for 43 (51\%) of these patients at baseline, and with treatment, remained the better-seeing eyes in 39 (91\%) patients after 3 years. Of the 81 never-treated eyes, 67 remained untreated up to the end of year 3 . The mean acuity of these never-treated eyes changed from $44.86 \mathrm{~L}$ to $43.48 \mathrm{~L}$ over 3 years.

\section{Central retinal thickness}

In first-treated eyes reaching month 36 follow-up, the CRT values at baseline were $423.30 \pm 115.38 \mu \mathrm{m}$ and then reduced to $325.01 \pm 79.10 \mu \mathrm{m}, 331.74 \pm 88.31 \mu \mathrm{m}$, and $325.08 \pm 97.62 \mu \mathrm{m}$ at 12,24 , and 36 months, respectively.

\section{Discussion}

We were able to reliably identify and complete abstraction of data from case notes of a consecutive cohort of patients from the baseline visit (first ranibizumab treatment) until a time point when they should have completed 3 years of follow-up. In total, 120 consecutive patients commenced therapy during a 9-month period, and 35 patients discontinued follow-up giving a 3-year attrition rate of $29 \%$ for our service. Only $3 \%$ required bilateral treatment from baseline, and $12 \%$ required second eye treatment within 3 years. Over the course of 3 years, this cohort of patients made a total of 3,188 visits and received 1,365 injections. From our data on service provision, we can deduce that for a population of 1 million, approximately 2,446 injections would be required in a 3 -year period. However, if fixed monthly injections were given, 7,282 injections would be required over a 3-year period for the new cases arising each year from a population of 1 million. With the introduction of newer agents for NV-AMD such as aflibercept, which may require less frequent injections, ${ }^{13,14}$ future audits may confirm a reduced burden on injection visits.

The treatment benefit for our cohort of patients after 3 years was visual stability. Overall, their mean change in acuity was only $-1.16 \mathrm{~L}$, and $78.2 \%$ of patients lost $<15 \mathrm{~L}$. To our knowledge, this is the first study to estimate the visual benefit to patients in terms of maintenance of the better-seeing eye with therapy in treatment-naive patients. In this cohort, as many as $51 \%$ of patients only received ranibizumab therapy in the better-seeing eye. Of these, $91 \%$ of patients continued to have better vision in their treated eyes after 3 years.

Our visual outcomes after 3 years are broadly comparable to those described in other "real-world" outcomes studies from the UK ${ }^{8,9}$ and other countries. ${ }^{11,15,16}$ Several methodological differences between these real-world studies are worth noting when comparing visual outcomes. Some studies ${ }^{9,11}$ converted Snellen visual acuities into $\log$ MAR scale, which has a tendency to over-estimate acuity, especially at the lower levels. A few studies had patients with variable follow-up duration and either excluded those with incomplete follow-up, making the sample unrepresentative at baseline, ${ }^{8}$ or did not take into account the impact of attrition when calculating change in visual acuity from baseline, such that the baseline mean acuity was calculated in a larger sample, and the final mean acuity was calculated from a smaller sample. ${ }^{9}$ In this study, we found that the exclusion of discontinued patients from the analysis can have a sizeable impact on visual outcome parameters. This negative impact of applying LOCF was also reported recently by Frennesson and Nilsson ${ }^{16}$ and Rasmussen et al. ${ }^{11}$ Excluding discontinued patients from the 
analysis and not applying LOCF can lead to overestimation of visual outcomes, as the commonest reason for discontinuing patients from follow-up was the development of end stage, "stable," macular damage. ${ }^{11,15}$ Studies which had a very high percentage of missing data or discharge rate can be difficult to interpret. Rasmussen et $\mathrm{al}^{11}$ reported a mean acuity of change from $0.24 \mathrm{~L}$ ( $\mathrm{n}=600$ eyes) to $0.18 \mathrm{~L}$ ( $\mathrm{n}=192$ eyes) after 4 years, and Ross et $\mathrm{l}^{17}$ reported an attrition from 406 eyes at 12 months to 198 eyes at 24 months due to insufficient follow-up data entry. These high attrition rates can obviously have a random and unpredictable impact on the estimation of visual outcome, even when LOCF is applied. Furthermore, it is important to note if a study had included patients who had prior PDT, ${ }^{11}$ as this can give rise to an underestimation of visual benefit from ranibizumab therapy.

The major strength of real-world studies is that they represent the true population that we treat in everyday settings. Randomized clinical trials are very good for demonstrating the maximum efficacy of a therapy but may not be representative of the general population due to the inevitable selection bias caused by the tendency to recruit patients with fewer comorbidities for clinical trials. In our study, we have been careful to be representative by analyzing consecutive patients and not excluding anyone from the baseline analysis because of subsequent incomplete follow-up data availability. We accounted for every patient that was discontinued and applied LOCF in the full-analysis set. Missing data was therefore due purely to discontinued patients due to our discontinuation policies and not due to incomplete data entry. This ensured a high level of representativeness of our study population. We had an attrition rate of $30 \%$ over 3 years, which can be tolerated when using the LOCF method. ${ }^{18,19}$ For comparing visual outcomes between sites, it is important to ensure the representativeness of the sample and the inclusion of discontinued patients in the final analysis using LOCF.

Like other real-world studies, our study also did not have best corrected visual acuity. When comparing with levels achieved in clinical trials, some allowance has to be made for an underestimation. Our sample size was also quite small as the study period we selected to give us 36 months of follow-up coincided with the time when we were recruiting into a clinical trial. As the inclusion criteria of the IVAN study was based on NICE guidelines, we do not think this created a selection bias for this study. A larger sample would have helped to generate narrower SDs on the estimates of visual acuity outcomes.

Rasmussen et $\mathrm{al}^{11}$ reexamined patients who had previously been discontinued from therapy due to disease inactivity and found that many patients continued to decline. This means that our method of applying LOCF to those we discharged may also give rise to an overestimation but probably not as much as would be caused by not applying LOCF and excluding them from analysis.

In conclusion, we have tried to provide an accurate evidence base of not only the visual benefit that can be gained from ranibizumab therapy by patients with NV-AMD over a 3-year period but also, for the first time, describe the actual burden of therapy in terms of hospital visits and injections for our patients. We surveyed a consecutive and representative sample of patients and took into account the impact of early discontinuation from follow-up on estimates of visual outcomes. For the first time, to our knowledge, we demonstrated a $91 \%$ rate of maintenance of stability in the better-seeing eye in the most desperate group of patients who were receiving unilateral treatment to their better-seeing eyes. We believe outcomes reported in real-world studies can be useful to emphasize the burden of disease and also to demonstrate the true benefit of therapy for patients outside the near-perfect world of the clinical trial setting. We also highlighted several pitfalls and inaccuracies that can occur when collecting data and auditing services and also in comparing visual and other outcome parameters for purposes of benchmarking, which may become increasingly important if we are to enter an era of making our clinical outcomes available for public scrutiny. ${ }^{20}$

\section{Disclosure}

The authors report no conflicts of interest in this work.

\section{References}

1. nice.org.uk [homepage on the Internet]. London: National Institute for Clinical Excellence; 2008 [updated May 2012]. Ranibizumab and pegaptanib for the treatment of age-related macular degeneration. Available from: http://publications.nice.org.uk/ranibizumab-and-pegaptanib-for-thetreatment-of-age-related-macular-degeneration-ta155. Accessed May 1, 2012.

2. Brown DM, Kaiser PK, Michels M, et al; ANCHOR Study Group. Ranibizumab versus verteporfin for neovascular age-related macular degeneration. N Engl J Med. 2006;355(14):1432-1444.

3. Rosenfeld PJ, Brown DM, Heier JS, et al; MARINA Study Group. Ranibizumab for neovascular age-related macular degeneration. $N$ Engl J Med. 2006;355(14):1419-1431.

4. CATT Research Group, Martin DF, Maquire MG, Ying GS, Grunwald JE, Fine SL, Jaffe GJ. Ranibizumab and bevacizumab for neovascular age-related macular degeneration. $N$ Engl J Med. 2011; 364(20):1897-1908.

5. IVAN Study Investigators, Chakravarthy U, Harding SP, Rogers CA, et al. Ranibizumab versus bevacizumab to treat neovascular age-related macular degeneration: one-year findings from the IVAN randomized trial. Ophthalmology. 2012;119(7):1399-1411.

6. Busbee BG, Ho AC, Brown DM, et al; HARBOR Study Group. Twelve-month efficacy and safety of $0.5 \mathrm{mg}$ or $2.0 \mathrm{mg}$ ranibizumab in patients with subfoveal neovascular age-related macular degeneration. Ophthalmology. 2013;120(5):1046-1056. 
7. Singer MA, Awe CC, Sadda S, et al. HORIZON: an open-label extension trial of ranibizumab for choroidal neovascularization secondary to age-related macular degeneration. Ophthalmology. 2012; 119(6):1175-1183.

8. Muniraju R, Ramu J, Sivaprasad S. Three-year visual outcome and injection rrequency of intravitreal ranibizumab therapy for neovascular agerelated macular degeneration. Ophthalmologica. 2013;230(1):27-33.

9. Pushpoth S, Sykakis E, Merchant K, Browning AC, Gupta R, Talks SJ. Measuring the benefit of 4 years of intravitreal ranibizumab treatment for neovascular age-related macular degeneration. Br J Ophthalmol. 2012;96(12):1469-1473.

10. Rofagha S, Bhisitkul RB, Boyer DS, Sadda SR, Zhang K; SEVEN-UP Study Group. Seven-year outcomes in ranibizumab-treated patients in ANCHOR, MARINA, and HORIZON: a multicenter cohort study (SEVEN-UP). Ophthalmology. 2013;120(11):2292-2299.

11. Rasmussen A, Bloch SB, Fuchs J, et al. A 4-year longitudinal study of 555 patients treated with ranibizumab for neovascular age-related macular degeneration. Ophthalmology. 2013;120(12):2630-2636.

12. Lalwani GA, Rosenfeld PJ, Fung AE, et al. A variable-dosing regimen with intravitreal ranibizumab for neovascular age-related macular degeneration: year 2 of the PrONTO Study. Am J Ophthalmol. 2009;148(1):43-58.

13. Thomas M, Mousa SS, Mousa SA. Comparative effectiveness of aflibercept for the treatment of patients with neovascular age-related macular degeneration. Clin Ophthalmol. 2013;7:495-501.

14. Schmidt-Erfurth U, Kaiser PK, Korobelnik JF, et al. Intravitreal aflibercept injection for neovascular age-related macular degeneration: ninety-six-week results of the VIEW studies. Ophthalmology. 2014;121(1):193-201.
15. Krüger Falk M, Kemp H, Sørensen TL. Four-year treatment results of neovascular age-related macular degeneration with ranibizumab and causes for discontinuation of treatment. Am J Ophthalmol. 2013;155(1):89-95.

16. Frennesson CI, Nilsson SE. A three-year follow-up of ranibizumab treatment of exudative AMD: impact on the outcome of carrying forward the last acuity observation in drop-outs. Acta Ophthalmol. Epub 2013 Mar 4.

17. Ross AH, Donachie PH, Sallam A, et al. Which visual acuity measurements define high-quality care for patients with neovascular age-related macular degeneration treated with ranibizumab? Eye (Lond). 2013;27(1):56-64.

18. Saha C, Jones MP. Bias in the last observation carried forward method under informative dropout. J Stat Plan Infer. 2009;139(2):246-255.

19. DiLoreto DA Jr, Bressler NM, Bressler SB, Schachat AP. Use of best and final visual acuity outcomes in ophthalmological research. Arch Ophthalmol. 2003;121(11):1586-1590.

20. rcseng.ac.uk [homepage on the Internet]. London: Royal College of Surgeons. RCS hails landmark publication of individual surgeons' outcomes data; 2013. Available from: http://www. rcseng.ac.uk/news/rcs-hails-landmark-publication-of-individualsurgeons2019-outcomes-data. Accessed June 28, 2013.
Clinical Ophthalmology

\section{Publish your work in this journal}

Clinical Ophthalmology is an international, peer-reviewed journa covering all subspecialties within ophthalmology. Key topics include: Optometry; Visual science; Pharmacology and drug therapy in eye diseases; Basic Sciences; Primary and Secondary eye care; Patient Safety and Quality of Care Improvements. This journal is indexed on

Submit your manuscript here: http://www.dovepress.com/clinical-ophthalmology-journal

\section{Dovepress}

PubMed Central and CAS, and is the official journal of The Society of Clinical Ophthalmology (SCO). The manuscript management system is completely online and includes a very quick and fair peer-review system, which is all easy to use. Visit http://www.dovepress.com/ testimonials.php to read real quotes from published authors. 